\title{
ESTUDIO EMPÍRICO DE LOS ENFOQUES DE APRENDIZAJE DE LOS ESTUDIANTES UNIVERSITARIOS EN FUNCIÓN DEL PERFIL DE SU TITULACIÓN (PROFUNDO VS SUPERFICIAL) ${ }^{1}$
}

\author{
EMPIRICAL RESEARCH ON UNIVERSITY STUDENTS' LEARNING \\ APPROACHES DEPENDING ON THE DEGREE UNDERTAKEN \\ (DEEP VS SUPERFICIAL)
}

\author{
F. Hernández, Ma . P. García y J. J. Maquilón \\ Universidad de Murcia
}

\begin{abstract}
RESUMEN
En la presente investigación se trata de estudiar, en primer lugar, a partir del análisis factorial los items que mejor definen los enfoques de aprendizaje -motivos y estrategias- de los estudiantes universitarios. En segundo lugar, se analiza la actuación que los estudiantes tienen en los items en cada una de las escalas en función del perfil de aprendizaje predominante en su titulación: superficial y profundo.

Los resultados muestran que los items de la subescala de motivo profundo se factorizan en un solo factor y los de estrategia en dos factores. En cambio, en el enfoque superficial, ambas subescalas se factorizan en tres factores. En relación con el segundo objetivo, se ha observado una coherencia entre el enfoque predominante en los estudiantes y el perfil del centro en el que estudian.
\end{abstract}

Palabras claves: Enfoques de aprendizaje, motivos y estrategias, perfil de la titulación universitaria.

\section{ABSTRACT}

In this research we use the factor analysis to analyse which items are the most representative in the Biggs' SPQ. Secondly, we also analyse the performance of students in the questionnaire to see whether there is any relationship between the learning approaches of their University course and the student performance.

1. Los enfoques de aprendizaje en el contexto de la Evaluación de la Calidad de las Universidades. Proyecto PB95-1002. Secretaría de Estado de Universidades, Investigación y Desarrollo. Dirección General de Enseñanza Superior. Subdirección General de Formación y Promoción del Conocimiento. Departamento MIDE. Facultad de Educación. e-mail: fhpina@um.es 
The results show that the scale for deep approach produce one main factor in motive subscale and two main factors in strategy scale. On the contrary, the scale for surface approach split in three main factors.

Concerning the second objective we find that there is a relationship between the faculties profile and the student performance.

Key words: Learning approaches, motives, strategies, student performance, faculty profile.

\section{Introducción}

La presente investigación se centra en el análisis de la calidad de los perfiles de aprendizaje de los estudiantes universitarios. Esta calidad varía en función de la percepción que van desarrollando a lo largo de su vida universitaria, del contexto y del ambiente en el que se desarrollan los procesos de enseñanza y de aprendizaje.

El estudio se encuentra enmarcado dentro la orientación cualitativa de la investigación sobre el aprendizaje, concretamente en la línea centrada en los enfoques de aprendizaje desarrollada por Marton, Entwistle y Biggs.

El término «enfoques de aprendizaje» fue utilizado por primera vez por Marton y Svensson -pertenecientes al grupo de Gotenburgo- en 1979. Estos autores, mediante estudios naturalistas, observaron que los alumnos/as podían adoptar un enfoque profundo o superficial al abordar ciertas tareas académicas.

Paralelamente a los trabajos de Marton y sus colegas, a finales de los años setenta y principios de los ochenta comienzan a aparecer otros trabajos en Gran Bretaña sobre modelos de aprendizaje dirigidos por Entwistle y Ramsden (1983) -Grupo de Edimburgo-. El objetivo principal de sus investigaciones era establecer cómo aprenden los estudiantes a partir de la información que éstos suministran.

Entwistle y su equipo, basándose en varias fuentes, elaboraron un modelo de aprendizaje y un cuestionario cuya finalidad era facilitar la obtención de una medida de los enfoques utilizados por los alumnos/as. Tras la definición y análisis conceptual de un número considerable de items, obtuvo un inventario que diferenciaba tres tipos de enfoques de aprendizaje:

a) Enfoque profundo/ aprendizaje por comprensión/ motivación intrínseca.

b) Enfoque superficial/ aprendizaje por operación/ motivación extrínseca/ miedo al fracaso; $y$

c) Enfoque estratégico/ método de estudio organizado/motivación de logro.

Casi en paralelo, los enfoques de aprendizaje han sido definidos por Biggs (1987) como los procesos de aprendizaje que emergen de la percepción que el estudiante tiene de la tarea académica influida por las características del individuo. El concepto implica, por consiguiente, elementos personales e institucionales que al interaccionar determinan los tipos de enfoque de aprendizaje adoptados por los estudiantes.

Biggs, en su modelo de aprendizaje -que a partir de 1989 denominó «Modelo 3P»- establece una clasificación de los enfoques de aprendizaje en tres niveles: enfoque superficial (SA), enfoque profundo (DA) y enfoque de alto rendimiento (AA), al tiempo que incorpora tanto los motivos como las estrategias de aprendizaje a cada uno de los mencionados enfo- 
ques, la predisposción que el sujeto tenga hacia un determinado enfoque será el modo con el que el estudiante logre el equilibrio en el sistema educativo tal y como lo percibe.

Para determinar los enfoques de aprendizaje de los alumnos/as, y por lo tanto la calidad del mismo, Biggs (1987) ha elaborado el Cuestionario de Procesos de Estudio (CPE), caracterizado por la incorporación de factores de presagio, proceso y producto y por la inclusión de seis subescalas correspondientes a los motivos y a las estrategias.

El trabajo que aquí se presenta tiene su punto de partida en un estudio anterior dónde se realiza la primera adaptación del cuestionario de Biggs al castellano (Hernández Pina, 1993, 1996).

A continuación presentamos en la tabla 1 el número de cada uno de los items del cuestionario (CPE) agrupados por escalas -enfoques- y subescalas. En el ANEXO I se puede encontrar una relación del contenido de todos los items del instrumento.

TABLA 1: Items del Cuestionario agrupados por enfoques.

\begin{tabular}{|c|c|c|c|c|c|c|}
\hline Escalas & \multicolumn{2}{|c|}{ Enfoque superficial } & \multicolumn{2}{c|}{ Enfoque profundo } & \multicolumn{2}{c|}{ Enfoque alto rendimiento } \\
\hline Subescalas & Motivo & Estrategia & Motivo & Estrategia & Motivo & Estrategia \\
\hline \multirow{4}{*}{$\begin{array}{c}\text { Número de } \\
\text { items }\end{array}$} & 1 & 4 & 2 & 5 & 3 & 6 \\
\cline { 2 - 7 } & 7 & 10 & 8 & 11 & 9 & 12 \\
\cline { 2 - 7 } & 13 & 16 & 14 & 17 & 15 & 18 \\
\cline { 2 - 7 } & 25 & 22 & 20 & 23 & 21 & 24 \\
\cline { 2 - 7 } & 31 & 34 & 36 & 29 & 27 & 30 \\
\hline
\end{tabular}

\section{Objetivos}

Los objetivos de la investigación se centran en:

1. Especificar los items que mejor definen los enfoques de aprendizaje -motivos y estrategias- de los estudiantes universitarios.

2. Determinar la actuación que los estudiantes tienen en los items en cada una de las escalas en función del perfil de aprendizaje predominante en su titulación: superficial y profundo.

\section{Metodología}

\section{Participantes}

La población de referencia de la investigación la constituyen los 14.661 alumnos/as de la Universidad de Murcia matriculados en dicha institución durante el año académico 
1996-1997 en primero y último año de carrera. La muestra -no aleatoria y con carácter accidental- ha quedado constituida por 3.861 alumnos/as de diecinueve titulaciones de la Universidad de Murcia -doce licenciaturas y siete diplomaturas-.

\section{Variables}

Las variables predictoras o independientes son las relativas al centro, el género, el curso (primero y último de carrera), la edad (con cinco niveles), titulación (Facultades y Escuelas Universitarias) y opción en que eligió la carrera que está cursando.

Como variables dependientes tenemos las puntuaciones obtenidas en los motivos, las estrategias y los enfoques de aprendizaje tal y como los operacionaliza el CPE.

\section{Instrumentos}

Para la recogida de información se ha utilizado el Study Process Questionnaire (SPQ) -Cuestionario sobre Procesos en el Estudio (CPE) - en versión española (Hernández Pina, 1993) de J.B. Biggs (1987), el cual, además del propio Cuestionario, incluye una hoja de respuestas y un manual.

\section{Procedimiento}

El procedimiento seguido en la investigación ha reunido las características propias de los estudios tipo encuesta o survey, siendo aplicado el Cuestionario, durante la hora de clase, a todos los alumnos/as que se encontraban en el aula en los grupos seleccionados el día y hora fijados para cada curso y centro, previa petición de permiso al profesor/a responsable.

\section{Análisis y Resultados}

El paquete estadístico utilizado para llevar a cabo el análisis de los datos ha sido SYSTAT, versión 5 de Wilkinson (1990) y el módulo complementario TESTAT para el análisis de items de prueba.

A continuación presentamos los análisis correspondientes a los objetivos planteados, así como los resultados y conclusiones de tales análisis.

OBJETIVO PRIMERO:

Especificar los items que mejor definen los enfoques de aprendizaje-motivos y estrategias- de los estudiantes universitarios

Tomando como punto de partida el análisis de componentes principales de primer orden realizado en otro estudio (Hernández Pina y otros, 1999) -en el que se obtuvieron diez factores con valor eigen mayor que uno, que explicaban el $47,88 \%$ de la varianza total-, he- 
mos determinado los items que mejor definen cada subescala atendiendo a las cargas factoriales que poseen, teniendo en cuenta que cargas que superan 0.71 las hemos considerado excelentes; entre 0.71 y 0.63 muy buenas; hasta 0.55 buenas; hasta 0.45 normales; hasta 0.32 pobres y por debajo de 0.30 eliminables (Comrey, 1973).

A partir de estos criterios, reseñamos a continuación -en la tabla 2- las cargas factoriales de cada uno de los items en función de la escala y subescala de origen.

TABLA 2: Cargas factoriales resultantes del análisis de primer orden.

\begin{tabular}{|r|c|c|c|c|c|c|c|c|c|c|c|}
\hline \multicolumn{4}{|c|}{ SA } & \multicolumn{5}{c|}{ DA } & \multicolumn{4}{c|}{ AA } \\
\hline \multicolumn{2}{|c|}{ SM } & \multicolumn{2}{|c|}{ SS } & \multicolumn{2}{c|}{ DM } & \multicolumn{2}{c|}{ DS } & \multicolumn{2}{c|}{ AM } & \multicolumn{2}{c|}{ AS } \\
\hline Item & Carga & Item & Carga & Item & Carga & Item & Carga & Item & Carga & Item & Carga \\
\hline 13 & 0.750 & 22 & -0.727 & 32 & 0.554 & 11 & 0.756 & 9 & 0.764 & 18 & 0.687 \\
\hline 7 & 0.726 & 4 & -0.697 & 2 & 0.528 & 23 & 0.753 & 15 & 0.694 & 12 & 0.674 \\
\hline 19 & 0.695 & 16 & 0.657 & 14 & 0.520 & 41 & 0.742 & 3 & 0.685 & 24 & 0.653 \\
\hline 37 & 0.607 & 40 & 0.607 & 20 & 0.488 & 29 & 0.686 & 33 & 0.683 & 42 & 0.639 \\
\hline 31 & 0.527 & 34 & 0.563 & 26 & 0.319 & 35 & 0.640 & 27 & -0.483 & 36 & 0.587 \\
\hline 1 & 0.385 & 10 & 0.452 & & & 5 & 0.581 & 39 & 0.403 & 30 & 0.448 \\
\hline 25 & -0.367 & 28 & 0.446 & & & 17 & -0.483 & & & & \\
\hline
\end{tabular}

A continuación se ha realizado un segundo análisis de componentes principales a partir de cada una de las subescalas.

TABLA 3: Cargas factoriales de los items del motivo superficial.

Subescala Motivo Superficial

\begin{tabular}{|c|c|c|c|}
\cline { 2 - 4 } \multicolumn{1}{c|}{} & \multicolumn{3}{c|}{ FACTORES } \\
\hline Items & I & II & III \\
\hline P13 & $\mathbf{0 . 7 6 5}$ & 0.090 & 0.079 \\
\hline P31 & $\mathbf{0 . 6 6 7}$ & 0.054 & -0.277 \\
\hline P37 & $\mathbf{0 . 6 3 6}$ & 0.041 & $\mathbf{0 . 4 3 1}$ \\
\hline P7 & 0.067 & $\mathbf{0 . 8 1 6}$ & -0.014 \\
\hline P19 & 0.073 & $\mathbf{0 . 8 0 8}$ & 0.052 \\
\hline P25 & -0.100 & 0.067 & $\mathbf{0 . 6 7 5}$ \\
\hline P1 & -0.108 & 0.031 & $-\mathbf{0 . 6 4 5}$ \\
\hline
\end{tabular}

\begin{tabular}{|l|r|r|r|r|}
\hline Eigen & 1.467 & 1.338 & 1.143 & \\
\cline { 1 - 3 } \% varianza & 20.954 & 19.112 & 16.325 & Total: $\mathbf{5 6 . 3 8 \%}$ \\
\hline
\end{tabular}


Analizada la subescala motivo superficial, se observa que los siete items que la forman se agrupan en tres factores. El primer factor agrupa a los items 13, 31 y 37; el segundo factor a los items 7 y 19; mientras que el tercer factor satura a los items 25 y 1 .

Los items saturados por el primer y tercer factor se refieren al pragmatismo de obtener una cualificación universitaria: Item 13 "Creo que los estudios superiores son imprescindibles para conseguir un trabajo estable y bien remunerado"; ítem 31 "Aunque me desagrada la idea de pasar varios años cursando una carrera, entiendo que el resultado final merece la pena" y el ítem 37 "Pienso que estoy en la universidad porque así consigo un mejor puesto de trabajo"; ítem 25 "Empleo poco tiempo en estudiar aquello que sé que no me va a salir en los exámenes" y el ítem 1 "Cuando tengo la oportunidad de elegir materiales de la carrera lo hago en función de mi satisfacción personal más bien que movido/a por intereses de mercado". El segundo factor satura items correspondientes al miedo al fracaso: el 7 "Me desanimo cuando saco malas notas y me pregunto cómo podré mejorarlas" y el 19 "Aunque estudio mucho para un examen, tengo la sensación de que puede no salirme bien".

TABLA 4: Cargas factoriales de los items de la estrategia superficial.

Subescala Estrategia Superficial

\begin{tabular}{|c|c|c|c|}
\cline { 2 - 4 } \multicolumn{1}{c|}{} & \multicolumn{3}{c|}{ FACTORES } \\
\hline Items & I & II & III \\
\hline P22 & $\mathbf{0 . 8 1 9}$ & 0.032 & 0.086 \\
\hline P4 & $\mathbf{0 . 8 1 1}$ & -0.011 & 0.064 \\
\hline P40 & -0.166 & $\mathbf{0 . 7 7 5}$ & 0.046 \\
\hline P34 & 0.237 & $\mathbf{0 . 7 2 3}$ & -0.016 \\
\hline P16 & 0.115 & -0.070 & $\mathbf{0 . 7 3 5}$ \\
\hline P28 & 0.079 & 0.318 & $\mathbf{0 . 5 5 4}$ \\
\hline P10 & 0.431 & 0.203 & -0.476 \\
\hline
\end{tabular}

\begin{tabular}{|l|r|r|r|l|}
\hline Eigen & 1.618 & 1.271 & 1.087 & \\
\hline \% varianza & 23.119 & 18.156 & 15.533 & Total: 56.81\% \\
\hline
\end{tabular}

Analizada la subescala estrategia superficial, se observa que los siete items que la forman se agrupan en tres factores. El primer factor agrupa a los items 22 y 4; el segundo factor a los items 40 y 34; mientras que el tercer factor satura a los items 16 y 28.

El factor I hace referencia al esfuerzo mínimo del alumno/a para aprobar: ítem 22 "En mis estudios me atengo a lo que específicamente me señalan en clase los profesores. Creo que no necesito hacer nada más" y el 4 "Realmente solo estudio los apuntes y lo que se me señala en clase. Entiendo que buscar información complementaria por mi cuenta es una pérdida de tiempo". El factor II se refiere a la consideración del profesor/a, por parte del alumno/a, muy por encima de éste: ítem 40 "Creo que los profesores saben bastante más que yo. Por eso considero que lo que dicen es importante y no valoro solamente mi propia opinión" y el ítem 34 "Prefiero aceptar las ideas de los profesores cuestionándolas sólo en circuns- 
tancias especiales". El factor III se refiere a que el alumno/a prefiere explicaciones del profesor/a desmenuzadas y relacionadas con la práctica: ítem 16 "Cuando tengo la posibilidad de elegir materias, me inclino por aquellas que se caracterizan por un contenido más factual (empírico) que teórico" y 28 "Como mejor aprendo es escuchando a aquellos profesores que dan la clase bosquejando con nitidez los puntos fundamentales”.

TABLA 5: Cargas factoriales de los items del motivo profundo.

Subescala Motivo Profundo

\begin{tabular}{|c|c|}
\cline { 2 - 2 } \multicolumn{1}{c|}{} & FACTOR \\
\hline Items & I \\
\hline P14 & $\mathbf{0 . 6 5 5}$ \\
\hline P20 & $\mathbf{0 . 6 4 0}$ \\
\hline P2 & $\mathbf{0 . 6 1 2}$ \\
\hline P26 & $\mathbf{0 . 6 0 4}$ \\
\hline P8 & $\mathbf{0 . 5 8 3}$ \\
\hline P38 & $\mathbf{0 . 5 3 5}$ \\
\hline P32 & $\mathbf{0 . 3 8 9}$ \\
\hline
\end{tabular}

\begin{tabular}{|l|r|}
\hline Eigen & 2.354 \\
\hline \% varianza & $\mathbf{3 3 . 6 3 3}$ \\
\hline
\end{tabular}

Analizada la subescala motivo profundo, se observa que los siete items que la forman se agrupan en un único factor, lo que muestra la homogeneidad de la subescala.

Los items 14, 20, 2 y 26 expresan el interés profundo de por qué se estudia, por ejemplo: ítem 14 "Todos los temas que tengo que estudiar me resultan interesantes una vez que profundizo en ellos"; e ítem 26 "Cuanto más estudio un tema más me absorbe". Los items 8,32 y 38 se refieren, en cambio, a aspectos filosóficos, como se observa en el ítem 32 "Creo que mi objetivo en esta vida es descubrir mi propia razón de ser y actuar estrictamente de acuerdo con dichos principios".

TABLA 6: Cargas factoriales de los items de estrategia profunda.

Subescala Estrategia Profunda

\begin{tabular}{|c|c|c|}
\cline { 2 - 3 } \multicolumn{1}{c|}{} & \multicolumn{2}{c|}{ FACTORES } \\
\hline Items & I & II \\
\hline P41 & $\mathbf{0 . 8 0 2}$ & 0.157 \\
\hline P11 & $\mathbf{0 . 7 8 5}$ & 0.118 \\
\hline P23 & $\mathbf{0 . 7 8 3}$ & 0.175 \\
\hline P5 & $\mathbf{0 . 5 6 5}$ & 0.180 \\
\hline P29 & 0.118 & $\mathbf{0 . 8 0 4}$ \\
\hline P35 & 0.155 & $\mathbf{0 . 7 6 7}$ \\
\hline P17 & 0.183 & $\mathbf{0 . 5 7 0}$ \\
\hline
\end{tabular}

\begin{tabular}{|l|r|r|l|}
\hline Eigen & 2.263 & 1.661 & \\
\hline \% varianza & 32.329 & 23.729 & Total: $\mathbf{5 6 . 0 6}$ \\
\hline
\end{tabular}


Analizada la subescala estrategia profunda, se observa que los siete items que la forman se saturan en dos factores. El primer factor satura a los items 41, 11, 23 y 5; y el segundo factor a los items 29, 35 y 17 .

Los items del primer factor muestran la utilización de cuatro estrategias profundas, ítem 41 "Cuando leo, relaciono todo lo nuevo con lo que ya sé sobre el tema"; ítem 11 "Cuando leo cosas nuevas, las relaciono automáticamente con lo que ya sé, y las veo bajo una nueva perspectiva"; ítem 23 "Intento relacionar lo que he aprendido en una materia con lo que sé de otra/s"; e ítem 5 "Cuando estudio, pienso en las aplicaciones de lo que aprendo a la vida real". Los items del segundo factor reflejan un claro interés y dedicación por lo que se estudia: ítem 29 "Encuentro toda temática nueva interesante y dedico tiempo a ampliarla buscando información adicional"; ítem 35 "Empleo bastante tiempo de mi tiempo libre profundizando en temas que me suscitan interés en diversas materias"; e ítem 17 "Cuando estudio algo, tengo que trabajarlo bastante para formarme una opinión personal al respecto y así quedar satisfecho".

TABLA 7: Cargas factoriales de los items del motivo de alto rendimiento.

\begin{tabular}{|c|c|c|}
\hline \multirow[b]{2}{*}{ Items } & \multicolumn{2}{|c|}{ FACTORES } \\
\hline & $\mathbf{I}$ & II \\
\hline P9 & 0.784 & 0.078 \\
\hline P15 & 0.769 & -0.004 \\
\hline P3 & 0.747 & 0.049 \\
\hline P33 & 0.631 & 0.334 \\
\hline P27 & 0.029 & 0.719 \\
\hline P39 & -0.010 & 0.610 \\
\hline P21 & 0.274 & 0.452 \\
\hline
\end{tabular}

\begin{tabular}{|l|r|r|l|}
\hline Eigen & 2.240 & 1.214 & \\
\hline \% varianza & 31.999 & 17.341 & Total: $\mathbf{4 9 . 3 4 \%}$ \\
\hline
\end{tabular}

Analizada la subescala de motivo de alto rendimiento, se observa que los siete items forman dos factores. El primero satura a los items 9, 15, 3 y 33; y el segundo a los items 27, 39 y 21.

Los items del primer factor hacen referencia a la ambición personal y a destacar en todo lo que se hace: ítem 9 "Siento un profundo deseo de destacar en todas las materias"; ítem 15 "Me considero una persona con ambición personal que quiere alcanzar el máximo en todo lo que hace"; ítem 3 "Mi objetivo es sacar las máximas calificaciones para así tener acceso a los mejores puestos de trabajo"; e ítem 33 "Lograr buenas notas lo veo como un juego competitivo en el que me gustaría jugar y ganar". El segundo factor satura tres items que hacen referencia a la competitividad: ítem 27 "Al elegir las materias lo hago pensando primeramente en la nota que pueda obtener"; ítem 39 "Entiendo que la sociedad es básicamente competitiva y que esto se refleja también en el sistema educativo"; e ítem 21 "Si me llegase el caso estaría dispuesto a sacrificar la popularidad inmediata que pudiera tener entre los compañeros con tal de tener éxito en mis estudios y en el ejercicio de mi carrera". 
TABLA 8: Cargas factoriales de los items de la estrategia de alto rendimiento. Subescala Estrategia Alto Rendimiento

\begin{tabular}{|c|c|c|}
\cline { 2 - 3 } \multicolumn{1}{c|}{} & \multicolumn{2}{c|}{ FACTORES } \\
\hline Items & I & II \\
\hline P18 & $\mathbf{0 . 7 2 8}$ & 0.176 \\
\hline P12 & $\mathbf{0 . 7 2 3}$ & 0.207 \\
\hline P42 & $\mathbf{0 . 7 1 8}$ & -0.056 \\
\hline P24 & $\mathbf{0 . 6 6 0}$ & 0.253 \\
\hline P30 & 0.018 & $\mathbf{0 . 7 3 1}$ \\
\hline P36 & 0.116 & $\mathbf{0 . 7 0 5}$ \\
\hline P6 & 0.182 & $\mathbf{0 . 5 6 9}$ \\
\hline
\end{tabular}

\begin{tabular}{|l|r|r|l|}
\hline Eigen & 2.064 & 1.496 & \\
\hline \% varianza & 29.491 & 21.370 & Total: $\mathbf{5 0 . 8 6 \%}$ \\
\hline
\end{tabular}

Analizada la subescala de estrategia de alto rendimiento, se observa que los siete items que la forman han sido agrupados en dos factores. El primer factor satura a los items 18, 12, 42 y 24 ; y el segundo al 30, 36 y 6.

El primer factor hace referencia a la organización del trabajo y a la predisposición al estudio: ítem 18 "Hago todas las tareas que me asignan cuanto antes"; ítem 12 "Estudio de una manera muy sistemática a lo largo del curso y reviso los apuntes con regularidad"; ítem 42 "Después de una clase releo los apuntes para asegurarme de que están claros y los entiendo"; e ítem 24 "Después de una clase releo los apuntes para asegurarme de que están claros y los entiendo". El segundo factor se refiere a la realización de tareas por parte del alumno: ítem 30 "Me pregunto a mí mismo/a sobre temas importantes hasta conseguir dominarlos perfectamente"; ítem 36 "Intento leer toda la bibliografía complementaria que el profesor señala para cada tema"; e ítem 6 "Resumo las lecturas señaladas por el profesor y las incluyo como parte de la materia en cuestión".

\section{OBJETIVO SEGUNDO:}

Determinar la actuación que los estudiantes tienen en los items en cada una de las escalas en función del perfil de aprendizaje predominante en su titulación: superficial y profundo

En el estudio ya mencionado (Hernández Pina y otros, 1999), las 19 titulaciones fueron clasificadas según su perfil predominante de aprendizaje, si bien para el análisis de este objetivo se han tomado solamente las cuatro primeras titulaciones que figuran con el porcentaje más alto en enfoque superficial -Graduado Social, Informática, Magisterio y Empresariales- y enfoque profundo -Filosofía, Medicina, Psicología y Fisioterapia- respectivamente. Añadir también que los items incluidos en el mencionado análisis son aquellos que factorialmente han resultado más relevantes (saturación superior a 0.30).

A continuación se ha calculado el porcentaje de respuestas que los estudiantes de cada titulación han dado en cada uno de los items. Para un mejor análisis, se han agrupado las cinco opciones de la escala que acompaña a cada pregunta, en dos intervalos. El primero 
agrupa las opciones 1 (nunca) y 2 (a veces), mientras que el segundo agrupa las opciones 4 (frecuentemente) y 5 (casi siempre), descartando la opción intermedia 3 (a menudo). En las tablas 9 y 10 se presentan por titulaciones los porcentajes acumulados en cada uno de los items de las subescalas, donde se reseña con el signo + aquel intervalo, cuya suma porcentual de opciones que aglutina, es superior; y con el signo - el intervalo restante. Si dicho porcentaje supera el $50 \%$ se ha añadido un asterisco.

En la tabla 9 se presenta -en función de las cuatro titulaciones seleccionadas con enfoque superficial- el mayor o menor porcentaje de los items de cada subescala. Dicho porcentaje ha quedado agrupado en los intervalos 1-2 ó 4-5, según la actuación de los estudiantes en cada uno de los items.

A continuación comentaremos el comportamiento de los alumnos/as -que cursan titulaciones con enfoque predominantemente superficial- en los items más relevantes que constituyen las seis subescalas del cuestionario.

\section{Subescala Motivo Superficial}

En la tabla 9 se observa que el comportamiento de los estudiantes en los items ha sido el esperado en las cuatro titulaciones ya que el mayor porcentaje se encuentra en el intervalo 4-5, excepto el ítem 25 que ha acumulado dicho porcentaje en los valores de la escala 1 y 2 en las titulaciones de Graduado Social, Magisterio y Empresariales. Este resultado es incoherente. Sin embargo, parece quedar justificado cuando comprobamos en el análisis de componentes principales que dicho ítem mostró una saturación negativa y el índice de fiabilidad también resultó bajo.

\section{Subescala Estrategia Superficial}

Los items 16, 40 y 28 parecen mostrar un porcentaje acumulado en los niveles de la escala 4 y 5 coherente con el perfil de cada titulación. Por el contrario, se aprecia un resultado incoherente en los items 22, 4, 34 y 10, respecto a la actuación de los estudiantes. La explicación de este resultado puede tener su origen en el análisis de componentes principales, donde los items 22 y 4 obtuvieron una saturación alta negativa, y los items 34 y 10 no obtuvieron una alta saturación. Además, estos cuatro items mostraron un índice de fiabilidad bastante bajo (inferior a 0.300 ).

\section{Subescala Motivo Profundo}

La actuación de los estudiantes ha sido la esperada en los items de esta subescala, excepto en el 32, en las cuatro titulaciones. En el análisis de componentes principales este ítem no saturó de forma única en el factor denominado DM, sino que lo hizo en el factor denominado DM-DS (Hernández Pina y otros, 1999). 
TABLA 9: Distribución porcentual en titulaciones con enfoque superficial.

2Titulaciones con enfoque superficial

\begin{tabular}{|c|c|c|c|c|c|c|c|c|c|c|}
\hline \multirow{3}{*}{$\begin{array}{l}\text { Enfo- } \\
\text { que }\end{array}$} & \multirow{3}{*}{$\begin{array}{c}\text { Sub } \\
\text { escala }\end{array}$} & \multirow{3}{*}{$\begin{array}{l}\mathrm{N}^{\circ} \\
\text { item }\end{array}$} & \multirow{2}{*}{\multicolumn{2}{|c|}{$\frac{\text { Grad social }}{\text { Opciones }}$}} & \multirow{2}{*}{\multicolumn{2}{|c|}{$\frac{\text { Informática }}{\text { Opciones }}$}} & \multirow{2}{*}{\multicolumn{2}{|c|}{$\frac{\text { Magisterio }}{\text { Opciones }}$}} & \multirow{2}{*}{\multicolumn{2}{|c|}{$\frac{\text { Empresariales }}{\text { Opciones }}$}} \\
\hline & & & & & & & & & & \\
\hline & & & $1-2$ & $4-5$ & $1-2$ & 4-5 & $1-2$ & $4-5$ & $1-2$ & $4-5$ \\
\hline \multirow{14}{*}{ SA } & \multirow{7}{*}{ SM } & 13 & - & $+*$ & - & $+*$ & - & $+*$ & - & $+*$ \\
\hline & & 7 & - & $+*$ & - & $+*$ & - & $+*$ & - & $+*$ \\
\hline & & 19 & - & $+*$ & - & $+*$ & - & $+*$ & - & $+^{*}$ \\
\hline & & 37 & - & $+*$ & - & $+*$ & - & + & - & $+*$ \\
\hline & & 31 & - & $+*$ & - & $+*$ & - & $+*$ & - & $+*$ \\
\hline & & 1 & - & $+*$ & - & $+*$ & - & $+*$ & - & $+*$ \\
\hline & & 25 & + & - & - & + & + & - & + & - \\
\hline & \multirow{7}{*}{ SS } & 22 & + & - & $+^{*}$ & - & $+^{*}$ & - & $+^{*}$ & - \\
\hline & & 4 & + & - & $+*$ & - & $+*$ & - & $+*$ & - \\
\hline & & 16 & - & + & - & $+*$ & - & $+*$ & - & $+*$ \\
\hline & & 40 & - & $+*$ & - & $+*$ & - & $+*$ & - & $+*$ \\
\hline & & 34 & + & - & $+*$ & - & $+*$ & - & + & - \\
\hline & & 10 & $+*$ & - & $+*$ & - & $+^{*}$ & - & $+^{*}$ & - \\
\hline & & 28 & - & $+^{*}$ & - & $+^{*}$ & - & $+*$ & - & $+^{*}$ \\
\hline \multirow{12}{*}{ DA } & & 32 & - & $+*$ & - & $+*$ & - & $+*$ & - & $+*$ \\
\hline & & 2 & + & - & + & - & $+^{*}$ & - & + & - \\
\hline & DM & 14 & + & - & + & - & $+^{*}$ & - & + & - \\
\hline & & 20 & $+*$ & - & $+*$ & - & $+^{*}$ & - & + & - \\
\hline & & 26 & + & - & + & - & $+*$ & - & $+*$ & - \\
\hline & \multirow{7}{*}{ DS } & 11 & - & + & - & $+*$ & - & + & - & $+*$ \\
\hline & & 23 & - & + & - & $+*$ & - & $+*$ & - & $+*$ \\
\hline & & 41 & - & + & - & $+*$ & - & $+^{*}$ & - & $+*$ \\
\hline & & 29 & $+*$ & - & $+*$ & - & $+^{*}$ & - & $+*$ & - \\
\hline & & 35 & $+*$ & - & $+*$ & - & $+*$ & - & $+*$ & - \\
\hline & & 5 & - & $+*$ & - & $+*$ & - & $+*$ & - & $+*$ \\
\hline & & 17 & - & + & + & - & + & - & + & - \\
\hline \multirow{12}{*}{ AA } & & 9 & + & - & + & - & $+^{*}$ & - & $+*$ & - \\
\hline & & 15 & - & + & - & $+*$ & - & + & - & $+*$ \\
\hline & & 3 & - & + & - & $+*$ & - & + & - & $+*$ \\
\hline & AM & 33 & $+*$ & - & + & - & $+^{*}$ & - & + & - \\
\hline & & 27 & $+*$ & - & $+*$ & - & $+^{*}$ & - & $+*$ & - \\
\hline & & 39 & - & $+*$ & - & $+*$ & - & $+*$ & - & $+^{*}$ \\
\hline & \multirow{6}{*}{ AS } & 18 & + & - & $+*$ & - & - & + & $+*$ & - \\
\hline & & 12 & + & - & $+*$ & - & $+^{*}$ & - & + & - \\
\hline & & 24 & $+*$ & - & $+*$ & - & $+^{*}$ & - & $+*$ & - \\
\hline & & 42 & - & $+*$ & - & + & - & $+^{*}$ & - & $+*$ \\
\hline & & 36 & $+^{*}$ & - & $+*$ & - & $+^{*}$ & - & $+*$ & - \\
\hline & & 30 & $+*$ & - & + & - & $+^{*}$ & - & $+*$ & - \\
\hline
\end{tabular}

\section{Subescala Estrategia Profunda}

Según se observa en la tabla 9, en los items 29, 35 y 17 los estudiantes han mostrado un comportamiento coherente con el perfil de la titulación a la que pertenecen, salvo en Graduado Social, donde en el ítem 17 la actuación de los alumnos/as ha sido contraria a la es- 
perada. En los items 11, 23, 41 y 5, se aprecia un comportamiento incoherente a lo que cabría esperar en todas las titulaciones. En el análisis de componentes principales, la saturación de estos items, ha sido medio-alta, excepto la del ítem 17 que resultó negativa. Los índices de fiabilidad también han resultado altos, no pudiendo concluir que estos items carezcan de validez de constructo y fiabilidad suficiente.

\section{Subescala Motivo Alto Rendimiento}

Los estudiantes han optado mayoritariamente por las opciones 1 y 2 en los items 9, 33 y 27, lo cual muestra un comportamiento coherente de acuerdo con el perfil de su titulación. Sin embargo, se aprecia una incoherencia en los items 15, 3, y 39, respecto a la actuación de los estudiantes. De estos tres últimos items, sólo el 39, obtuvo una saturación baja en el análisis de componentes principales, y un índice de fiabilidad también bajo.

\section{Subescala Estrategias Alto Rendimiento}

La respuesta de los estudiantes en los items de esta subescala ha resultado bastante coherente en las cuatro titulaciones, ya que el mayor porcentaje se encuentra en el intervalo 12, excepto el ítem 42, que lo acumula en el intervalo 4-5 en todas las titulaciones, y el ítem 18, en donde los estudiantes se comportan de la misma forma en la titulación de Magisterio. Puesto que tanto el ítem 42 como el 18 obtuvieron una alta saturación en el análisis de componentes principales y también un alto índice de fiabilidad, no podemos decir que sendos items estén afectados por su validez de constructo y fiabilidad.

En la tabla 10 se presenta -en función de las cuatro titulaciones seleccionadas con enfoque profundo- el mayor o menor porcentaje de los items de cada subescala, Dicho porcentaje ha quedado agrupado en los intervalos 1-2 ó 4-5, según la actuación de los estudiantes en cada uno de los items.

A continuación comentaremos el comportamiento de los alumnos/as -que cursan titulaciones con enfoque predominantemente profundo- en los items más relevantes que constituyen las seis subescalas del cuestionario.

\section{Subescala Motivo Profundo}

La actuación de los estudiantes ha sido la esperada en los items de esta subescala, excepto en el ítem 26, en las titulaciones de Psicología y Fisioterapia. Así pues, los estudiantes se han comportado coherentemente con el perfil de su titulación, puesto que el mayor porcentaje de respuestas se ha agrupado en los niveles 4 y 5 de la escala en cada una de las preguntas. El hecho de que los alumnos se hayan comportado de forma incoherente ante el ítem 26 en dos de las titulaciones queda justificado al observar la baja saturación obtenida por este ítem en el análisis de componentes principales. 
TABLA 10: Distribución porcentual en titulaciones con enfoque profundo.

Titulaciones con enfoque profundo

\begin{tabular}{|c|c|c|c|c|c|c|c|c|c|c|}
\hline \multirow{3}{*}{$\begin{array}{c}\text { Enfo- } \\
\text { que }\end{array}$} & \multirow{3}{*}{$\begin{array}{c}\text { Sub } \\
\text { escala }\end{array}$} & \multirow{3}{*}{$\begin{array}{l}\mathrm{N}^{\circ} \\
\text { item }\end{array}$} & \multicolumn{2}{|c|}{ Filosofía } & \multicolumn{2}{|c|}{ Medicina } & \multicolumn{2}{|c|}{ Psicología } & \multicolumn{2}{|c|}{ Fisioterapia } \\
\hline & & & \multicolumn{2}{|c|}{ Opciones } & \multicolumn{2}{|c|}{ Opciones } & \multicolumn{2}{|c|}{ Opciones } & \multicolumn{2}{|c|}{ Opciones } \\
\hline & & & $1-2$ & $4-5$ & $1-2$ & $4-5$ & $1-2$ & $4-5$ & $1-2$ & $4-5$ \\
\hline \multirow{14}{*}{ SA } & \multirow{7}{*}{ SM } & 13 & + & - & - & $+*$ & - & $+*$ & - & $+*$ \\
\hline & & 7 & $+*$ & - & - & $+*$ & - & $+*$ & - & $+^{*}$ \\
\hline & & 19 & $+*$ & - & - & $+*$ & - & $+*$ & - & $+*$ \\
\hline & & 37 & $+*$ & - & + & - & $+^{*}$ & - & + & - \\
\hline & & 31 & - & $+*$ & - & $+*$ & - & $+*$ & - & $+*$ \\
\hline & & 1 & - & $+*$ & - & $+^{*}$ & - & $+*$ & - & $+*$ \\
\hline & & 25 & $+*$ & - & $+*$ & - & $+*$ & - & $+^{*}$ & - \\
\hline & \multirow{7}{*}{ SS } & 22 & $+*$ & - & $+^{*}$ & - & $+^{*}$ & - & $+*$ & - \\
\hline & & 4 & $+*$ & - & $+*$ & - & $+^{*}$ & - & $+^{*}$ & - \\
\hline & & 16 & $+*$ & - & - & $+^{*}$ & - & $+^{*}$ & - & $+^{*}$ \\
\hline & & 40 & - & $+*$ & - & $+*$ & - & $+*$ & - & $+*$ \\
\hline & & 34 & $+*$ & - & + & - & $+^{*}$ & - & + & - \\
\hline & & 10 & $+*$ & - & $+*$ & - & $+^{*}$ & - & $+*$ & - \\
\hline & & 28 & - & + & - & $+*$ & - & $+*$ & - & $+^{*}$ \\
\hline \multirow{12}{*}{ DA } & & 32 & - & $+*$ & - & $+*$ & - & $+*$ & - & $+*$ \\
\hline & & 2 & - & + & - & $+*$ & - & $+*$ & - & + \\
\hline & DM & 14 & - & $+*$ & - & $+*$ & - & + & - & + \\
\hline & & 20 & - & $+*$ & - & $+*$ & - & $+*$ & - & + \\
\hline & & 26 & - & + & - & + & + & - & + & - \\
\hline & \multirow{7}{*}{ DS } & 11 & - & $+*$ & - & $+*$ & - & $+*$ & - & $+^{*}$ \\
\hline & & 23 & - & $+*$ & - & $+*$ & - & $+*$ & - & $+*$ \\
\hline & & 41 & - & $+*$ & - & $+*$ & - & $+^{*}$ & - & $+^{*}$ \\
\hline & & 29 & + & - & + & - & $+^{*}$ & - & $+^{*}$ & - \\
\hline & & 35 & - & + & $+*$ & - & $+*$ & - & $+*$ & - \\
\hline & & 5 & - & $+*$ & - & $+*$ & - & $+^{*}$ & - & $+^{*}$ \\
\hline & & 17 & - & + & - & $+*$ & - & + & - & $+^{*}$ \\
\hline \multirow{12}{*}{ AA } & & 9 & $+*$ & - & - & + & $+^{*}$ & - & $+^{*}$ & - \\
\hline & & 15 & - & + & - & $+^{*}$ & - & + & - & $+*$ \\
\hline & & 3 & $+*$ & - & - & $+*$ & + & - & - & $+^{*}$ \\
\hline & AM & 33 & $+*$ & - & $+*$ & - & $+*$ & - & $+^{*}$ & - \\
\hline & & 27 & $+*$ & - & $+*$ & - & $+^{*}$ & - & $+^{*}$ & - \\
\hline & & 39 & - & $+*$ & - & + & $+^{*}$ & - & - & $+*$ \\
\hline & \multirow{6}{*}{ AS } & 18 & $+*$ & - & - & $+*$ & + & - & + & - \\
\hline & & 12 & $+*$ & - & - & + & + & - & - & + \\
\hline & & 24 & $+*$ & - & - & + & $+*$ & - & + & - \\
\hline & & 42 & + & - & - & $+*$ & - & $+^{*}$ & - & $+^{*}$ \\
\hline & & 36 & + & - & $+*$ & - & $+^{*}$ & - & $+*$ & - \\
\hline & & 30 & $=$ & $=$ & + & - & $+^{*}$ & - & + & - \\
\hline
\end{tabular}

\section{Subescala Estrategia Profunda}

En los items 11, 23, 41, 5 y 17 los estudiantes acumulan el mayor porcentaje en el nivel alto de la escala -opciones 4 y 5-, lo cual ratifica la coherencia de la actuación de estos alumnos/as de acuerdo con el perfil profundo de la titulación a la que pertenecen. Sin em- 
bargo, se observa que en las titulaciones de Medicina, Psicología y Fisioterapia el mayor porcentaje de estudiantes se acumula en el nivel bajo de la escala-opciones 1 y 2 - en los items 35 y 29. Estos items, tanto en el análisis de componentes principales como en el análisis de la fiabilidad, obtuvieron resultados medio-altos, lo cual nos hace pensar que la actuación contraria se deba a que dichos items no resultaron ser factorialmente puros de estrategia profunda (DS), sino que fueron saturados por un factor combinación de estrategia profunda y estrategia de alto rendimiento (DS-AS).

\section{Subescala Motivo Superficial}

De las cuatro titulaciones con perfil de enfoque profundo, solamente Filosofía ha resultado fiel a la obtención del mayor porcentaje de estudiantes en el nivel inferior de la escala en casi todos los items correspondientes a la motivación superficial -items 13, 7, 19, 37 y 25-. Excepcionalmente, los items 31 y 1 han acumulado el mayor porcentaje de respuestas en el extremo superior de la escala. En el caso de las otras tres titulaciones, lejos de lo que cabría esperar, el mayor porcentaje se ha acumulado en el extremo superior de la escala en los siguientes items: 13, 7, 19, 31 y 1 . Los únicos items que han sido fiel reflejo de la actuación de los estudiantes en motivo superficial y el perfil profundo que ostentan, han sido los items 37 y 25. Estos items, como se puede comprobar en el ANEXO, son un fiel reflejo de comportamientos antisuperficiales.

\section{Subescala Estrategia Superficial}

Los estudiantes han mostrado un comportamiento coherente con el perfil de su titulación en los items 22, 4, 34 y 10. Solamente en el caso de Filosofía, a la anterior lista se añade el ítem 16. El resto de los items -40, 28 y 16- han resultado con un mayor porcentaje de respuestas acumulado en el nivel superior de la escala.

\section{Subescala Motivo Alto Rendimiento}

Al analizar la actuación de los estudiantes en lo items de esta subescala, parece que cabría esperar que los mayores porcentajes se acumularan en los niveles altos de la escala. Como se puede observar en la tabla 10, esto no ha ocurrido así, puesto que solamente el ítem 15 ha acumulado el mayor porcentaje en el nivel superior de la escala en las cuatro titulaciones. En los items 33 y 27 los estudiantes muestran un comportamiento incoherente en todas las titulaciones al obtenerse los porcentajes más altos en las opciones 1 y 2 de la escala. Los mismos resultados se obtienen en el ítem 9 en las titulaciones de Filosofía, Psicología y Fisioterapia y también en el ítem 39 en la titulación de Psicología. Esta dispersión de la actuación de los estudiantes en los diversos items nos da una idea de la baja validez de constructo en esta subescala. 


\section{Subescala Estrategias Alto Rendimiento}

Al igual que ocurría con el motivo de alto rendimiento, también cabría esperar que la actuación de los estudiantes en los items de esta subescala fuese en la dirección de obtener los mayores porcentajes en los niveles más altos de la escala. Como se puede observar en la tabla 10, los resultados obtenidos en esta ocasión también han sido bastante contrarios a los esperados. Por lo tanto, a la dispersión observada en el motivo de alto rendimiento hay que añadir la de estrategia del mismo enfoque, donde se observa como en Filosofía, Psicología y, en menor grado, Fisioterapia, los estudiantes se acumulan en mayor porcentaje en el nivel inferior de la escala, mientras que en Medicina lo hacen en el nivel superior de la escala.

\section{Conclusiones}

Una vez analizados los datos de la investigación y determinados los resultados de la misma, finalizamos nuestro estudio explicitando las siguientes conclusiones:

1. De todos los items incluidos en el Cuestionario CPE, algunos han resultado tener una baja carga factorial -items 8(DM), 38(DM), 21(AM) y 6(AS)- y otros incluso carga negativa -items 25(SM), 22(SS), 4(SS), 17(DS) y 27(AM)-. Estos items, tal y como está haciendo actualmente el autor del cuestionario original (Biggs, 2000; comunicación personal) están siendo sometidos a una profunda revisión.

2. Del análisis de componentes principales llevado a cabo dentro de cada subescala, se observa que la de motivo profundo ha resultado ser la más homogénea, dado que todos los items han quedado saturados bajo un mismo factor. Las subescalas estrategia profunda y motivo y estrategia de alto rendimiento lo han hecho en dos factores. En cambio, las dos subescalas de enfoque superficial -motivo y estrategia- han resultado las más dispersas al factorizarse sus items en tres factores.

3. La actuación que han mostrado los estudiantes procedentes de las titulaciones con enfoque superficial ha resultado altamente coherente con la subescala motivo. Esta coherencia viene avalada por su actuación en motivo profundo, donde el mayor porcentaje se encuentra acumulado en el nivel inferior de la escala.

4. En el caso de la actuación de los estudiantes procedentes de titulaciones con perfil profundo, se observa que solamente los estudiantes de Filosofía acumulan el mayor porcentaje en el nivel inferior de la escala en los items de motivo superficial, lo que indica la alta coherencia del comportamiento de estos alumnos/as. Sin embargo, en el caso de Medicina, Psicología y Fisioterapia se observa que el mayor porcentaje de respuestas de los estudiantes se encuentra en el nivel superior de la escala. Cabría preguntarse: ¿Podrían ser este hecho coherente con las demandas de la Institución?

5. Como última conclusión nos gustaría resaltar que este trabajo constituye, en gran medida, un intento de llevar a cabo un diagnóstico contextualizado de cómo los estudiantes universitarios abordan su estudio y su aprendizaje, para, en una fase posterior, poder diseñar programas de intervención específicos de acuerdo con el perfil de la titulación y las actuaciones concretas y específicas de los estudiantes en los items de las distintas subescalas. 


\section{Bibliografía}

Biggs, J. B. (1987). Student approaches to learning and studying. Melburne. Australian Council for Educational Research.

Biggs, J. B. (1989a). Approaches to the enhancement to tertiary teaching. Higher Education Reseach and Development, 9, 7-25.

Biggs, J. B. (1989b). Does learning about learning help teacher with teaching? Psychology and the Tertiary teacher. Supplement to the Gazette, 36 (1), 21-34. University of Hong Kong.

Comrey, A. L. (1973). A first course in factor analysis. New York: Academic Press.

Entwistle y Ramsden (1983). Understanding Student learning. London: Croom Helm.

Hernández Pina, F. (1993). Concepciones en el estudio del aprendizaje de los estudiantes universitarios. Revista de Investigación Educativa, 22, $2^{\circ}$ semestre, 117-150

Hernández Pina, F. (1996). La evaluación de los alumnos en el contexto de la evaluación de las universidades. Revista de Investigación Educativa, 14 (2), 25-50.

Hernández Pina, F. y otros (1999). Los enfoques de aprendizaje en el contexto de la evaluación de la calidad de las universidades. Proyecto PB95-1002 subvencionado por la DGICYT.

Marton y Svenson (1979). Conceptions of research in student learning. Higher Education, 8, pp. 471486.

Wilkinson, L. (1990). SYSTAT. Manual del usuario. Ilinois: Evanston.

Fecha de recepción: 15-VI-00

Fecha de revisión: 10-III-01

Fecha de aceptación: 16-X-01 